\title{
Evaluation of Antifungal Activity of Curcumin against Aspergillus flavus
}

\author{
Anwesha Gitika ${ }^{1 *}$, Rajashree Mishra ${ }^{1}$, Susen Kumar Panda ${ }^{2}$, \\ Chinmoy Mishra ${ }^{3}$ and Pravas Ranjan Sahoo ${ }^{4}$
}

${ }^{1}$ Department of Veterinary Microbiology, ${ }^{2}$ Department of Veterinary Pathology, ${ }^{3}$ Department of Animal Breeding and Genetics, ${ }^{4}$ Department of Veterinary Biochemistry, College of Veterinary Science and Animal Husbandry, OUAT, Bhubabeswar, India

*Corresponding author

\section{A B S T R A C T}

\section{Keywords}

Curcumin, Aspergillus species, Antifungal action, Poultry

Article Info

Accepted:

17 June 2019

Available Online:

10 July 2019
The present study was undertaken to study the antifungal effect of curcumin and other synthetic antifungal agents against Aspergillus species isolated from poultry feed. During this study poultry feeds collected from poultry farm of Odisha Veterinary College. Various concentrations of curcumin like $25 \mathrm{mg} / \mathrm{disc}, 50 \mathrm{mg} / \mathrm{disc}, 100 \mathrm{mg} / \mathrm{disc}$ and $200 \mathrm{mg} / \mathrm{disc}$ were prepared and were tested by disc diffusion method. In this method antifungal activity of various agents like ketoconazole, voriconazole, itraconazole, clotrimazole, Amphotericin $\mathrm{B}$, miconazole, caspofungin, fluconazole were tested and its zone of inhibition was found to be approximately $\geq 18 \mathrm{~mm}, \geq 15 \mathrm{~mm}, \geq 16 \mathrm{~mm}, \geq 18 \mathrm{~mm}, \geq 7 \mathrm{~mm}, \geq 10 \mathrm{~mm}, \geq 18 \mathrm{~mm}$ and $\geq 10 \mathrm{~mm}$ respectively. It has been revealed that curcumin treated Aspergillus species showed lesser zone of inhibition than synthetic antifungals like ketoconazole, voriconazole, itraconazole, clotrimazole Amphotericin B, caspofungin but in comparison to fluconazole and miconazole curcumin shows larger zone of inhibition. The antifungal sensitivity pattern shows that curcumin $(200 \mathrm{mg} / \mathrm{disc})$ has got the highest potential of antifungal action against $A$. flavus and $A$. fumigatus in comparison to synthetic antifungals.

\section{Introduction}

Aspergillosis is the major infectious fungal disease of poultry caused by the fungal genus Aspergillus. This disease is most commonly caused by Aspergillus fumigatus and less commonly by A. flavus, A. niger, A. nidulans and A. terreus (Arne et al., 2011; Girma et al., 2016).

Aspergillus is ubiquitous and the common soil saprophyte reported in avian species domestic as well as wild birds (Atlaman, 1997). It also affects human beings and other animals. Avian species are highly susceptible due to the anatomical and physiologic feature of the respiratory system of the bird. The small nonexpanding lungs and nine air sacs are the predominant focus of infection (Nardoni et al., 2006). These organisms can sustain on substantial environmental stress condition including the humid environment of the poultry shed, improper ventilation system and damaged egg of hatcheries. These filamentous fungi grow on organic material in warm environment. Fungal proliferation and 
sporulation resulted in production of conidiophores from feed, faecal material and soil in suitable conditions. These conidia spread in the air and are possibly inhaled and loaded in the respiratory tract. Aspergillosis usually occurs in young birds as acute aspergillosis, which causes high morbidity and mortality. Older avian species exhibit the sporadic form with lesser mortality (Kunkle et al., 2003). Aspergillus species enter the egg shell and infect the embryo which may die or hatch with progressive lesions (Bauk, 1994)

Curcumin is the major curcuminoid found in turmeric and is well known for its multiple pharmacological and biological properties (Gupta et al., 2013; Zhou et al., 2011; Shen and $\mathrm{Ji}, 2012$ ). It possesses anti-inflammatory, antibacterial, antidiabetic, hepatoprotective, anticancer and anti-fungal properties (Chattopadhyay et al., 2004; Kohli et al., 2005 and Teiten et al., 2010). Dietary inclusion of $222 \mathrm{mg} / \mathrm{kg}$ curcuminoids had reduced the adverse impact of aflatoxin $\mathrm{B} 1$ on serum total protein, albumin and $\gamma$ - Glutamyl transferase activity (Gowda et al., 2009). The antifungal activity of curcumin has low side effects for that reason it shows synergy with synthetic fungicides. Curcumin affects Aspergillus growth and its morphology (Ferreira et al., 2013). Antifungal activity of curcumin is experimented and it has shown its effect against Aspergillus flavus, $A$. parasiticus, Fusarium moniliforme and Penicillium digitatum (Jayaprakasha et al., 2001). Antibacterial and antifungal action in poultry is less documented. So in the present study an attempt has been carried out to evaluate action of curcumin against fungus particularly Aspergillus species.

\section{Materials and Methods}

\section{Sample collection}

In the present study a total of 12 numbers of feed samples were collected from instructional poultry farm of Odisha veterinary College and also from different poultry houses starting from brooder house, broiler house and layer house during the period from December 2018 to May 2019. Four samples from each house were collected and screened for presence of various mycological agents with special reference to Aspergillus species. These samples were collected in plastic bottles and were immediately stored a $-20^{\circ} \mathrm{C}$ until analysis.

\section{Mycological analysis}

Isolation of fungus was done as per the methods of Sivakumar et al., (2014). The poultry feeds without additive or preservative were taken for analysis otherwise it might affect fungal growth. Poultry feeds were coarsely ground before analysis. $10 \mathrm{~g}$ of feed was weighed into $250 \mathrm{ml}$ of flask and equivalent amount of distilled water was added. Flasks were incubated at $37^{\circ} \mathrm{C}$ for up to 2 weeks and examined daily for fungal growth (Fig. 1). Distilled water was added to triplicate flasks to maintain the moisture content at day 6 and 14 of the incubation period.

\section{Identification of the mould}

After observing the fungal growth in the flask, the fungal conidia, mycelium were carefully removed and inoculated into sabourods dextrose agar and potato dextrose agar. The plates were incubated at $37^{\circ} \mathrm{C}$ for 48-72 hours. Identification of the fungus was done by observing colony morphology with reference to colour, size of mycelium and nature of colony. Lactophenol cotton blue staining and Gram staining were carried out as per routine procedure.

\section{Antifungal susceptibility test-}

Curcumin was obtained from Hi-media, Mumbai and Curcumin disc was prepared as 
per the method of Esimone et al., (2006). Various concentrations of curcumin discs were prepared which includes $25 \mathrm{mg}$, $50 \mathrm{mg}$, $100 \mathrm{mg}$ and $200 \mathrm{mg} /$ disc respectively. Muller Hinton Agar (MHA) obtained from M/S HiMedia Laboratories Ltd. Mumbai was employed for the in-vitro antifungal sensitivity test of fungal isolates as per the commonly used disc diffusion method Bauer et al., (1966) and the zone of inhibition was recorded. The antifungal discussed are ketoconazole $(10 \mathrm{mcg})$, voriconazole $(1 \mathrm{mcg})$, itraconazole $(30 \mathrm{mcg})$, clotrimazole $(10 \mathrm{mcg})$, Amphotericin $\mathrm{B}(20 \mathrm{mcg})$ miconazole

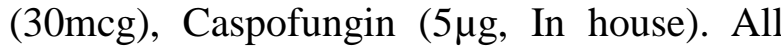
the antifungal disks were obtained from HiMedia, Mumbai, India.

\section{Results and Discussion}

In the present study Aspergillus flavus was the predominant isolates followed by $A$. niger, $A$. fumigatus, $A$. nidulans and $A$. terreus. The result is similar to that of the study of Ahmed et al., (2017).A total of 12 randomly selected isolates were identified by studying the colony morphology on Sabouraud dextrose agar and potato dextrose agar plates. Macroscopically Aspergillus flavus produces velvety or wooly yellowish green colonies, A,niger produced jet black conidia cottony, velvety or powdery colonies were produced by $A$. fumigatus. Velvety buff to yellow colour colonies in case of A.nidulus and cinnamon buff colour colonies in case of
A.terreus were observed (Fig. 2a, 2b). On lactophenol cotton blue staining Aspergillus flavus, Aspergillus niger, Aspergillus fumigatus, Aspergillus terreus were identified (Fig. 3a, 3b, 3c). Club shaped vesicle with uniseriate conidia was identified as A. fumigatus, biseriate phialides with long conidiophores in case of A.flavus, biseriate elliptical conidia with upward sweeping appearance in case of A.terreus, biseriate with profuse conidiation in case of A.niger and biseriate hemispherical vesicles were observed in case of A.nidulus under microscope. In gram staining, it was found that the fungal organism and the hyphae were stained purple (Fig. 4). This mycological study is in agreement with the study of Klich (2002) and Mc Clenny (2005).

On analysis of antifungal susceptibility pattern produced by various antifungals and curcumin (at various concentrations), it was found that almost all Aspergillus species $(n=12 / 12)$ were found sensitive to curcumin at the concentration of $200 \mathrm{mg} /$ disc. In case of Aspergillus flavus, synthetic antifungals like itraconazole, AmphotericinB ketoconazole, voriconazole, clotrimazole, caspofungin, miconazole and fluconazole with minimum inhibitory zone of 22, 8, 21, 16, 20, 2111 and $10 \mathrm{~mm}$ respectively whereas curcumin shows zone of inhibition of $\geq 10 \mathrm{~mm}$ diameter at $200 \mathrm{mg} / \mathrm{disc}$ and $<10 \mathrm{~mm}$ in case of $100 \mathrm{mg} /$ disc and 50mg/disc (Table 1 and 2).

Table.1 Prevalence and isolation of different Aspergillus sp. from poultry feed samples

\begin{tabular}{|l|c|c|}
\hline \multicolumn{1}{|c|}{ Name of the fungal isolates } & No. of isolation & Isolation (\%) \\
\hline Aspergillus flavus & $12 / 12$ & 100 \\
\hline Aspergillus niger & $9 / 12$ & 75 \\
\hline Aspergillus fumigatus & $8 / 12$ & 67 \\
\hline Aspergillus nidulans & $6 / 12$ & 50 \\
\hline Aspergillus terreus & $5 / 12$ & 42 \\
\hline
\end{tabular}


Table.2 Comparative study of synthetic antifungal agents with Curcumin disc (at various concentration)

\begin{tabular}{|c|c|c|c|c|c|c|c|c|c|c|c|c|}
\hline \multirow{3}{*}{$\begin{array}{l}\text { Name } \\
\text { fungal } \\
\text { isolates }\end{array}$} & \multicolumn{12}{|c|}{ Zone of inhibition in $\mathrm{mm}$} \\
\hline & \multicolumn{4}{|c|}{ ofCurcumin(CUR) } & \multirow[t]{2}{*}{ ITR } & \multirow[t]{2}{*}{ AMB } & \multirow[t]{2}{*}{ KT } & \multirow[t]{2}{*}{$\mathrm{VCZ}$} & \multirow[t]{2}{*}{ MIC } & \multirow[t]{2}{*}{ FLC } & \multirow[t]{2}{*}{$\mathrm{CC}$} & \multirow[t]{2}{*}{ CAS } \\
\hline & $25 \mathrm{mg}$ & $50 \mathrm{mg}$ & $100 \mathrm{mg}$ & $200 \mathrm{mg}$ & & & & & & & & \\
\hline A.flavus & $10 \pm 0.05$ & $11 \pm 0.00$ & $12 \pm 0.04$ & $14 \pm 0.1$ & $22 \pm 0.5$ & $8 \pm 0.56$ & $21 \pm 0.7$ & $16 \pm 0.0$ & $511 \pm 0$ & $210 \pm 0.05$ & $20 \pm 0$ & $221 \pm 0.00$ \\
\hline A.niger & $10 \pm 0 . .01$ & $10 \pm 0.04$ & $11 \pm 0.00$ & $12 \pm 0.00$ & $19 \pm 1.0$ & $8 \pm 0.6$ & $19 \pm 0.8$ & $20 \pm 0.0$ & $512 \pm 0$ & $11 \pm 0.04$ & $18 \pm 0$ & $018 \pm 0.05$ \\
\hline A.fumigatus & $9 \pm 0.00$ & $11 \pm 0.02$ & $12 \pm 0.02$ & $13 \pm 0.04$ & $18 \pm 0.7$ & $9 \pm 1.0$ & $20 \pm 0.65$ & $15 \pm 0.0$ & $010 \pm 0$ & $512 \pm 1.00$ & $21 \pm 0$ & $219 \pm 0.01$ \\
\hline A.nidulans & $10 \pm 0.05$ & $10 \pm 0.00$ & $11 \pm 0.03$ & $11 \pm 0.12$ & $19 \pm 0.5$ & $8 \pm 1.2$ & $18 \pm 0.75$ & $20 \pm 1.0$ & $11 \pm 0$ & $010 \pm 0.00$ & $19 \pm 0$ & $218 \pm 0.75$ \\
\hline A.terreus & $11 \pm 0.04$ & $12 \pm 0.01$ & $11 \pm 0.05$ & $13 \pm 0.57$ & $17 \pm 1.2$ & $7 \pm 0.8$ & $20 \pm 1.0$ & $19 \pm 1.2$ & $11 \pm 0$ & $513 \pm 0.00$ & $18 \pm 0$ & $020 \pm 0.02$ \\
\hline
\end{tabular}

Fig.1 Isolation of fungus from feed

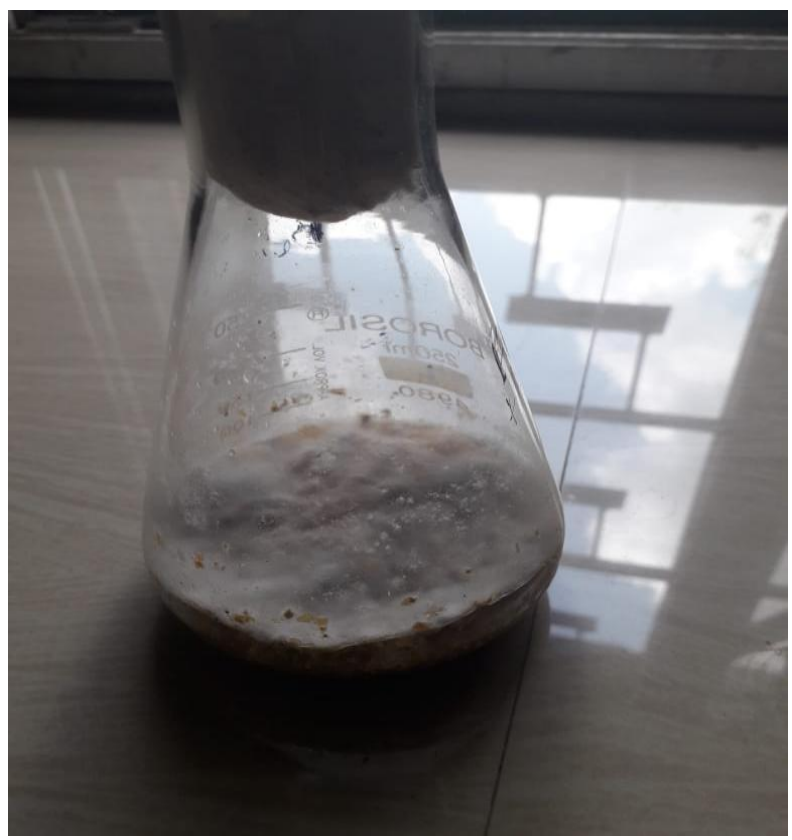

Fig.2(a) showing mixed culture of fungus

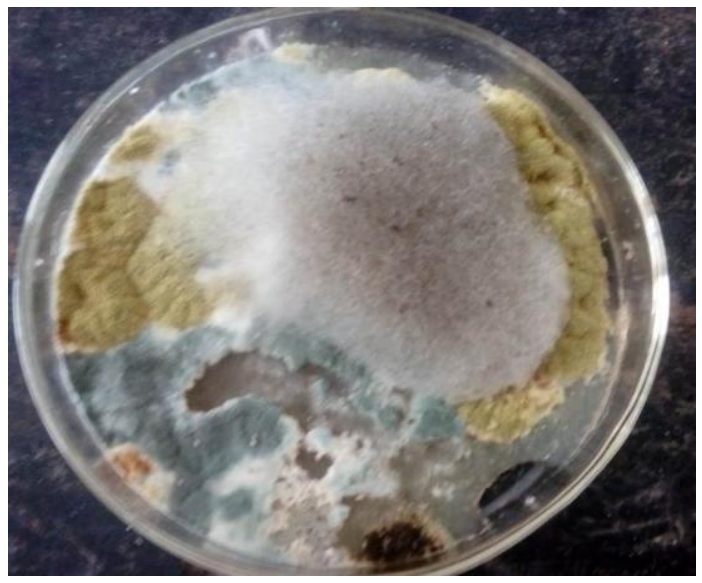

Fig.2(b) showing pure culture

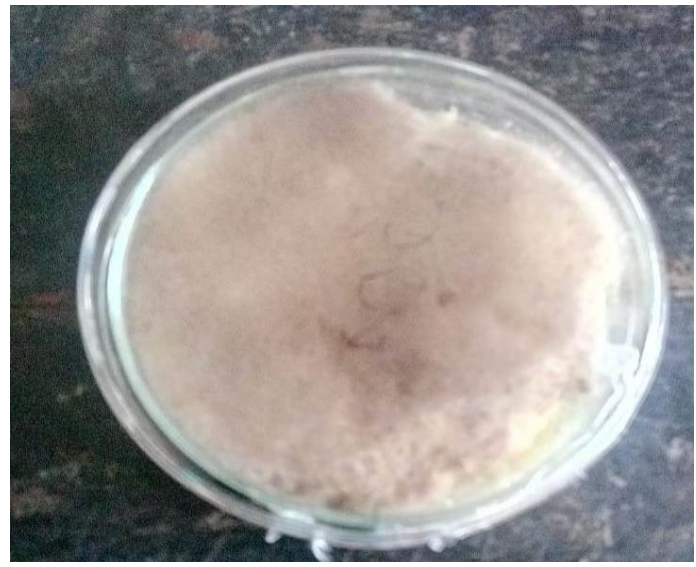


Fig.3 showing lactophenol cotton blue staining of fungal culture

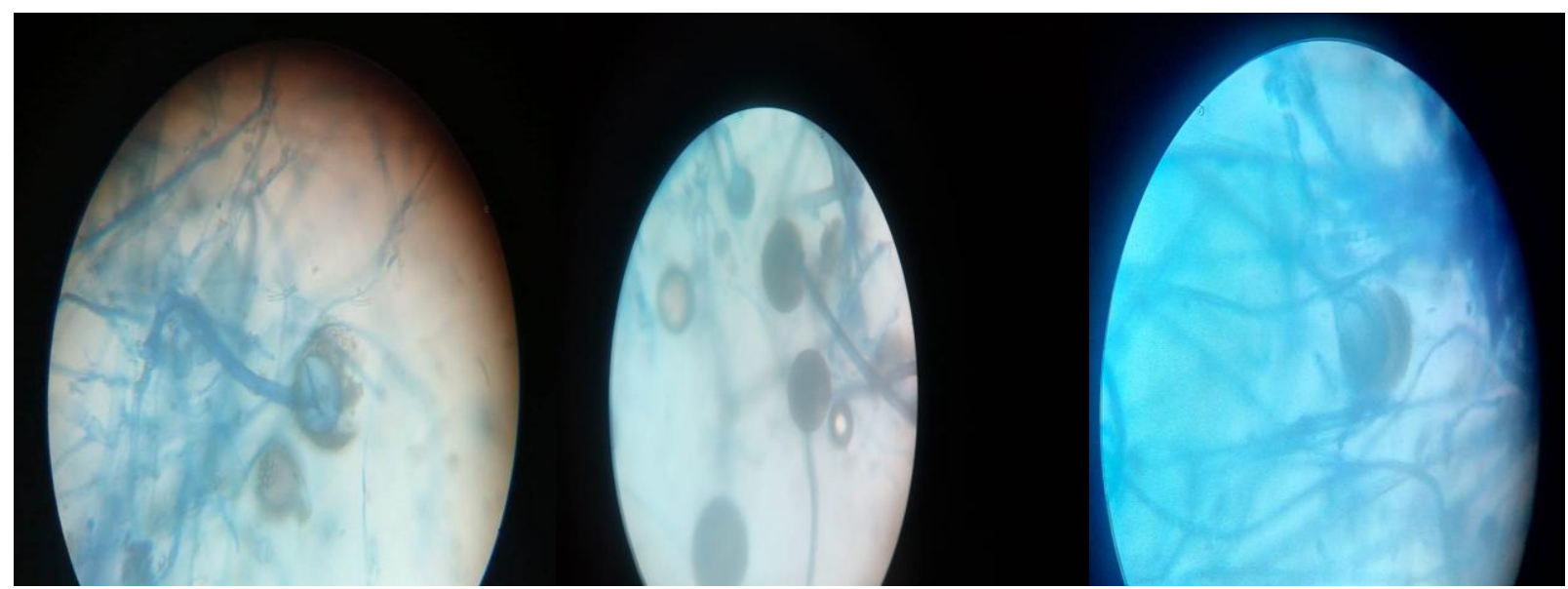

Fig.4 Gram staining of Aspergillus species

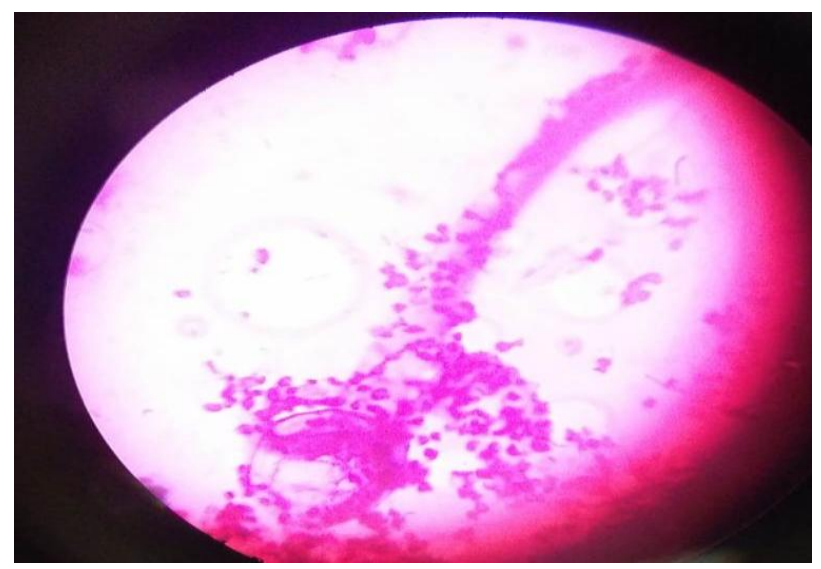

In case of Aspergillus niger synthetic antifungals like itraconazole, AmphotericinB, ketoconazole, voriconazole, clotrimazole, caspofungin miconazole, fluconazole showed minimum inhibitory zone of 19,8,19,20,12,18, 12 and $11 \mathrm{~mm}$ respectively where as curcumin shows zone of inhibition of approximately $\geq$ $10 \mathrm{~mm}$ diameter. In case of Aspergillus fumigatus synthetic antifungals like itraconazole, AmphotericinB, ketoconazole, voriconazole, clotrimazole, caspofungin miconazole, fluconazole showed minimum inhibitory zone of $18,9,20,15,21,19,10$ and $12 \mathrm{~mm}$ respectively whereas curcumin shows zone of inhibition of $\geq 9 \mathrm{~mm}$ diameter. In case of Aspergillus nidulans synthetic antifungal like itraconazole, AmphotericinB, ketoconazole, voriconazole, clotrimazole, caspofungin miconazole, fluconazole showed minimum inhibitory zone of $19,8,18,20,19$, 18,11 and $10 \mathrm{~mm}$ respectively whereas curcumin shows zone of inhibition of $\geq 10 \mathrm{~mm}$ diameter. In case of Aspergillus terreus synthetic antifungals like itraconazole, AmphotericinB, ketoconazole, voriconazole, clotrimazole, caspofungin miconazole, fluconazole showed minimum inhibitory zone of $17,7,20,19,18,20,11$ and $13 \mathrm{~mm}$ respectively whereas curcumin shows zone of inhibition of $\geq 11 \mathrm{~mm}$ diameter. The present study revealed that Amphotericin B is resistant to almost all Aspergillus species 
isolated from poultry feed is in agreement with study of Ramesh et al., (2013).In the present study, curcumin shows better zone of inhibition than that of miconazole, fluconazole and amphotericinB. More investigation is required in order to use curcumin as an potential antifungal agent for poultry.

\section{References}

Ahmed D. A; Al-Khafaji N.J and. Ahmed L T (2017) Isolation and Molecular Identification of Aspergillus spp. Collected from Different Sources of Animals Feed Int.J.Curr.Microbiol.App.Sci 6(6): 1792-1797.

Arne, P., S. Thierry, D. Wang, M. Deville and G. Le Loch et al., 2011. Aspergillus fumigatus in poultry. Int. J. Microbiol., 10.1155/2011/746356.

Atlaman RB (1997) Avian Medicine and Surgery. Saunders, Philadelphia, USA, 89-92.

Bauer AW., Kirby WM and Sherris JC..(1996) Antibiotic susceptibility testing by a standardized single disk method, American Journal of Clinical Pathology, 45: 493- 49.

Bauk L, Ritchie BW, Harrison GJ, Harrison LR (1994) Mycosis. In: Avian medicine principle and application. Pp. 174-180.

Chattopadhyay I., Biswas K., Bandyopadhyay U., Banerjee RK. (2004) Turmeric and curcumin: Biological actions and medicinal applications. Current Science, 87: 44-53.

Esimone C. O., Iroha, I. R., Ibezim, E. C Okeh, C. O. and Okpana, E., (2006) In vitro evaluation of the interaction between tea extracts and penicillin $G$ against staphylococcus aureus African Journal of Biotechnology 5(11):10821086.

Ferreira F D., Kemmelmeier C., Arrot'eia
CC. (2013) Inhibitory effect of the essential oil of Curcuma longa L. and curcumin on aflatoxin production by Aspergillus flavus Link, Food Chemistry, 136(2): 789-793.

Girma G., Abebaw M., Zemene M., Mamuye Y and Getaneh G. (2016) A Review on Aspergillosis in Poultry, Journal of veterinary science and technology, 7:1-5.

Gowda, N.K.S., Ledoux, D.R., Rottinghaus, G.E., Bermudez, A.J., and Chen, Y.C. (2009) Antioxidant efficacy of curcuminoids from turmeric (Curcuma longa L.) powder in broiler chicken fed diets containing aflatoxin B-1. Br. J. Nutr. 102: 1629-1634.

Gupta SC., Kismali G, Aggarwal BB (2013). Curcumin, a component of turmeric: from farm to pharmacy. Biofactors, 39: $2-13$.

Jayaprakasha, G. K., Negi, P. S., Anandharamakrishnan, C. and Sakariah, K. K. (2001). Chemical composition of turmeric oil - a byproduct from turmeric oleorsin industry and its inhibitory activity against different fungi. Z. Naturforsch., C, 56, 40-44.

Klich M. (2002) Identification of Common Aspergillus species. Utrech. The Netherlands: Centraal bureeau voor Schimmelculture.

Kohli K, Ali J, Ansari MJ, Raheman Z. (2005). Curcumin: A natural antiinflammatory agent. Indian $\mathrm{J}$ Pharmacol, 37:141-47.

McClenny N. (2005) Laboratory detection and identification of Aspergillus species by microscopic observation and culture. Journal of Medical Mycology., 1: 125128.

Nardoni S, Ceccherelli R, Rossi G, Mancianti F. 2006. Aspergillosis in Larus cachinnans micaellis: survey of eight cases. Mycopathologia, 161: 317-321.

Kunkle, R.A., Y.M. Saif, H.J. Barnes and 
Glisson, J. R (2003) “Aspergillosis,”in Diseases of Poultry, 11: 883-895.

Ramesh S., Kumar S.R, Balaji M.R. (2013) Antibiotic susceptibility of Aspergillus Spp. Isolated from contaminated food sources Der Pharmacia Lett. 5(6):179183.

Shen L., Ji F.S. 2012. The pharmacology of curcumin: Is it the degradation products? Trends in Molecular Medicine, 18(3): 138-144.

Sivakumar V.K. (2014) Isolation, characterization and growth optimization of toxicogenic molds from different animal feeds in Tamilnadu. Int.J.Curr.Microbiol.App.Sci 3(9):430445

Teiten MH, Eifes S, Dicarto M and Diederich M (2010). Curcumin the paradigm of a multi-target natural compound with applications in cancer prevention and treatment. Toxins., 2: 128-162.

Zhou H, Beevers CS and Huang S (2011). The targets of curcumin. Curr. Drug Targets, 12: 332-34.

\section{How to cite this article:}

Anwesha Gitika, Rajashree Mishra, Susen Kumar Panda, Chinmoy Mishra and Pravas Ranjan Sahoo. 2019. Evaluation of Antifungal Activity of Curcumin against Aspergillus flavus. Int.J.Curr.Microbiol.App.Sci. 8(07): 2323-2329. doi: https://doi.org/10.20546/ijcmas.2019.807.284 